\title{
ARTICLE
}

\section{Probing the Gene eXpression Database for candidate genes}

\author{
Maurice AM van Steensel, J Celli, JH van Bokhoven and HG Brunner \\ Department of Human Genetics, University Hospital Nijmegen, The Netherlands
}

\begin{abstract}
We report on a strategy for the identification of candidate genes for multiple malformation syndromes using expression data available in public databases. The basis for this pilot study was the assumption that, for a multiple malformation syndrome, the expression pattern of the causative gene should at least cover the organs or tissues affected by the syndrome. Twenty malformation syndromes were selected from the OMIM and defined by three to five main symptoms. These key symptoms were translated into anatomical terms that were used to query the Gene eXpression Database (GXD). The searches covered $65 \%$ of the database and yielded an average of 16 candidate genes per syndrome. Of these, $23 \%$ were ubiquitously expressed or housekeeping genes. Further database evaluation of these potential candidate genes was based on positional information and on information from mouse knockouts. In a first experiment, the correct gene was identified as a candidate in four of seven syndromes for which the causative gene is already known. In addition, this strategy identified new candidate genes for disorders for which the genetic basis is unknown. We identified candidate genes for the Walker-Warburg, DOOR, C, scalp-ear-nipple and oculocerebral hypopigmentation syndromes. Our results suggest that it may ultimately be feasible to identify disease genes by probing gene expression databases with simple syndrome descriptions.
\end{abstract}

Keywords: candidate gene; gene expression; database; syndrome; Internet

\section{Introduction}

Four main strategies for identifying human disease genes are often distinguished, namely functional cloning, positional cloning, positional candidate, and position-independent candidate strategies. In functional cloning, information about the presumed cellular function is used to identify the unknown disease gene. The gene product is already known and the gene coding for it can quickly be identified. This approach has been

Correspondence: HG Brunner MD, Department of Human Genetics 417, Radboud University Hospital Nijmegen, PO Box 9101, 6500 HB Nijmegen, The Netherlands. Tel: +31243614017; Fax: +31243540488; E-mail: h.brunner@antrg.azn.nl

Received 10 March 1999; revised 26 August 1999; accepted 1 September 1999 useful for those diseases in which well-known metabolic pathways or structural proteins are affected, such as phenylketonuria, alkaptonuria and haemophilia. ${ }^{1-3}$ In positional cloning, the gene is isolated on the basis of its chromosomal localisation, without prior information about its function. To achieve this, first the localisation of the disease gene must become known. This is done by means of linkage analysis using extended families. Next, genetic and physical maps of the chromosome area are constructed and potential genes in the region identified. Finding a disease-associated mutation is required to prove which of the genes in the region is the disease gene.

Positional cloning is gradually being superseded by the positional candidate approach. Thanks to the increasing volume of gene sequences, either partial or 
complete, deposited in public databases, candidate genes can often be found by database searches, targeting the area where a disease gene has been localised. In this way, the efforts involved in cloning and analysing large pieces of chromosomal DNA can often be avoided. When a disease gene is mapped to a specific chromosomal segment, additional criteria are useful to prioritise the different positional candidates. Among these criteria is the expression pattern of the positional candidate genes. ${ }^{4}$ A different approach from the positional cloning and positional candidate strategies is that of the position-independent candidate. For example, genes that are expressed in a single tissue can be considered as candidate genes for those inherited disorders in which only that tissue is affected. Examples are the collagen genes in the chondrodysplasias, ${ }^{5}$ retinaspecific genes in inherited blindness ${ }^{6}$ and cochleaspecific genes in deafness. ${ }^{7}$ Natural or induced mutations in model organisms such as the mouse or similar syndromes in humans for which the gene is already known $^{8,9}$ may further suggest a gene for a particular disorder. Human multiple malformation syndromes are defined by specific recurring patterns of abnormalities in tissues of different embryonic origin. In analogy to the candidate approach for single-tissue disorders, genes that have an expression pattern matching the syndromal pattern of abnormalities can be considered as candidates. To our knowledge, a generalised approach that compares gene expression data with syndrome-specific patterns of organ or tissue involvement has not been attempted. Here we report on our experience with such a strategy for gene identification based on matching gene-expression patterns with syndrome-specific patterns of organ involvement.

\section{Methods}

\section{Syndrome Definition}

In order to search databases for candidate genes, a syndrome must first be translated into an expression pattern. To define those areas in which gene expression is critical for the development of the syndrome, its defining features should be separated from rare or nonspecific signs. This is a central problem in syndromology for which many solutions have been proposed. ${ }^{10}$ These solutions, however, have not been incorporated into clinical syndromology practice nor in the available syndrome databases. Ideally, one would use a database that attaches weights to signs and symptoms based on their sensitivity and specificity relative to other syndromes. The London Dysmorphology Database ${ }^{11}$ (LDDB) does define a syndrome as a pattern, but does not distinguish essential from non-essential symptoms. The (P)OSSUM databases $^{12,13}$ can allocate a weight to symptoms, but as this must be done by the user, this does not obviate the problem. We chose to circumvent this problem by asking two experienced clinical geneticists to define a set of 20 syndromes by three to five main symptoms. The symptoms were chosen from the listings in the LDDB, as this is the most comprehensive database to date. A third clinical geneticist decided where the first two disagreed on a particular core symptom.

\section{Definition of Search Terms}

To search gene expression databases, the phenotypic abnormalities of the syndrome must be translated into search terms. At the time this work was undertaken, the expression databases that were available (such as the Genome Database (GDB) and the Gene eXpression Database (GXD)) were based entirely on literature abstracts. Therefore we defined the most commonly used developmental terms in the literature and used these in our searches. It should be noted that the newer GXD versions do have standardised expression terms.

To define the most commonly used terms, the 1997 issues of three major developmental biology journals (Genes and Development, Mechanisms of Development, Development) were examined for possible search terms. Both abstracts and the body of text of approximately 540 papers were evaluated. This resulted in a list of 1775 terms. Several of these were synonymous terms (for example, genito-urinary and urogenital). The 1775 terms were checked for occurrence in 3000 MedLine abstracts which had been selected using 'embryo' and 'development' as keywords. A simple shell script was written to count the terms and run on a PC running RedHat Linux 5.0-5.2, kernel versions 2.0.34-2.0.36. Terms that occurred at a frequency greater than 15 per 1000 articles were selected for use as search terms. A total of 153 terms was thus selected, less than $1 \%$ of the original list. These terms were arranged in a hierarchical look-up table which closely follows the branching structure of the LDDB feature list. Most of the headings of the LDDB feature list are matched by at least one term in the thesaurus. The search terms are grouped under organ systems headings as syndrome features in the LDDB. Syndrome features selected by the expert panel could thus be translated into developmental search terms by selecting the appropriate organ system heading and choosing the terms describing the affected tissue.

\section{Selection of a Suitable Database}

The starting point for the candidate gene selection procedure described here is the hypothesis that the gene which causes a multiple congenital malformation syndrome is expressed in the tissues affected in that particular syndrome. To find candidate genes based on this assumption, a database must contain searchable expression information for human genes. No such databases are at present available. We therefore used mouse data as a second-best approach. There are many databases available on the mouse as a model organism, ${ }^{14}$ but only the Gene eXpression Database (GXD), maintained by the Jackson Laboratories (www.informatics.jax.org), contains systematically ordered expression data that can be searched using anatomical or functional terms. This database was selected to examine the feasibility of candidate identification based on expression patterns.

\section{Candidate Gene Selection}

To find candidate genes for a specific syndrome, between two and four search terms were used to query the Gene 
eXpression Database. Compound searches, wildcarding and the use of regular expressions (a pattern that is matched against a string) are not allowed in this database. Each new search term requires a new query. The result of a query is a list of hyperlinked genes and their associated literature references, encoded in HTML (HyperText Markup Language). The genes in the listings are expressed in the tissue described by the search term. Candidate genes for the syndrome are those that are expressed in all tissues affected in the syndrome, ie are present in all listings. A shell-script was written to compare the downloaded files and select those genes that were present in each of the listings downloaded for that syndrome.

\section{The Pilot Study}

In order to evaluate the strategy outlined above, a pilot study was conducted. Twenty malformation syndromes were selected from the OMIM (Online Mendelian Inheritance in Man) database ${ }^{16}$ by querying it with 'syndrome' and picking OMIM numbers from the list at random. Syndromes for which the gene is already known, chromosome abnormalities and metabolic diseases were excluded. Syndromes were selected if the component abnormalities involved two or more anatomically distinct organ systems. The selected syndrome features were translated into search terms by use of the look-up table. A potential problem is that genes may be indexed under synonymous terms. Using only one term from the list of possible synonyms would give rise to many potential candidate genes being missed. To evaluate the magnitude of this problem, searches were made either using 'blanket terms', ie terms describing the organ system in the broadest sense, or by a list of synonyms, for example, 'limb' $v s$ 'hand + digit + ulna + radius'. Separate sets were compiled either of 'blanket terms' or of sets of synonyms. These terms were then entered in the GXD. The resulting HTML-encoded listings were downloaded and saved for later evaluation. Candidate gene listings were prepared by using the shell script that identifies genes which arise in all searches. In order to evaluate the potential for this approach to uncover disease genes, a set of seven syndromes for which the molecular basis is already known was also examined.

\section{Results}

\section{Characteristics of the Search Terms}

The 29 blanket search terms (Table 1 ) used to describe the total of 20 syndromes in the initial pilot study together recovered $1596(65 \%)$ of 2467 genes in the GXD (number as determined on 15 June 1998). One third of the database, 886 genes, was not accessed by this set of terms. This could be due to our limited selection of terms, but other causes such as missing expression data also seem possible. To clarify this, the abstracts of 40 genes not among those retrieved by the 29 blanket terms were evaluated (genes listed in Table 2). It turned out that 18 out of 40 genes either had no abstract or an abstract without anatomical search terms. Of another 18 genes, the abstracts contained terms which are not among the 29 terms selected in the initial study, but are in the main list of 153 terms. Of the remaining four genes, one had a term not present in either list and one entry had been removed from the database. A small number (two out of 40) genes was associated with abstracts which described a different

Table 1 Syndromes selected at random from OMIM and their description in expression patterns

\begin{tabular}{lll}
\hline Syndrome (No. of genes returned) & MIM number & Translation/search terms \\
\hline C-syndrome (20) & 211750 & Cranium, digit, brain \\
Cerebro-costo-mandibular syndrome (13) & 117650 & Rib, palate \\
FG syndrome (3) & 305450 & Cranium, ear, brain, hindgut \\
Wolcott-Rallison syndrome (1) & 226980 & Vertebra, limb, pancreas \\
Weill-Marchesani syndrome (8) & 277600 & Eye, tooth, digit \\
Branchio-oculo-facial syndrome (15) & 113620 & Branchial, eye, skeleton \\
Cerebro-oculo-facial-skeletal syndrome (5) & 214150 & Brain, skull, eye, digit \\
Costello syndrome (61) & 218040 & Heart, brain, skin \\
Oculocerebral-hypopigmentation syndrome (12) & 257800 & Brain, eye, pigment \\
DOOR syndrome (5) & 220500 & Ear, kidney, digit \\
Johnson-McMillin neuro-ectodermal syndrome (32) & 147770 & Brain, skin, tooth \\
Walker-Warburg syndrome (56) & 236670 & Muscle, eye, brain \\
Megalocornea-mental retardation syndrome (18) & 249310 & Cornea, brain \\
Nance-Horan syndrome (50) & 302350 & Eye, tooth \\
OFD type 1 (1) & 311200 & Brain, palate, tongue, digit \\
OFD type 3 (1) & 258850 & Tongue, digit, brain \\
Opitz-Frias syndrome (7) & 145410 & (O)esophagus , genital \\
Scalp-ear-nipple syndrome (4) & 181270 & Skin, ear, mammary \\
Senior-Loken syndrome (35) & 266900 & Retina, kidney \\
Varadi-Papp syndrome (4) & 277170 & Cerebellum, oral, digit
\end{tabular}

'both 'oesophagus' and 'esophagus' were used as search terms and the results concatenated since both terms return different sets of genes. 
Table 2 Properties of genes not found using 29 blanket search terms

\begin{tabular}{|c|c|c|c|}
\hline Gene & Abstract & Gene & Abstract \\
\hline Hp1a & no terms & Canp3 & no abstract \\
\hline Hsd17b4 & terms in list & $\mathrm{Cd} 3 \mathrm{e}$ & terms in list \\
\hline Iap1 & terms in list & Cea10 & terms in list \\
\hline Lamp1 & terms in list & Cebpa-rsl & no terms \\
\hline Uty & no terms & Cmkbrll2 & terms in list \\
\hline Abl & terms in list & Dabl & no terms \\
\hline Ar & terms in list & Dmahp & no terms \\
\hline Fgdl & no terms & Err2 & about other gene \\
\hline Lbxl & terms in list & Gnal2 & no terms \\
\hline Mif & terms in list & Gnao & terms in list \\
\hline Rnu6 & no terms & Gnaz & terms in list \\
\hline $\mathrm{Clk} 4$ & no terms & H2-T17 & no terms \\
\hline Rab6kifl & no terms & Has3 & no terms \\
\hline Tbxl4 & none & Hbb-y & about other gene \\
\hline Etv6 & terms in list & Mage-rsl & no terms \\
\hline Etv5 & no terms & Semak & removed from $\mathrm{db}$ \\
\hline Fgfr3-ps & no terms & Tcra-C & terms in list \\
\hline Finl3 & no terms & Zfx & terms in list \\
\hline
\end{tabular}

'Terms in list': terms found in abstracts can also be found in extended list of 153 search terms.

gene from the one indexed. Assuming that our sample of 40 genes analysed is representative of the 886 genes that were not retrieved, the following calculations can be made. Of all the genes currently in the GXD database, some $65 \%$ are accessible by a set of 29 simple blanket-type search terms and this increases to $81 \%$ if an extensive list of 153 (including several synonymous terms) is used. Of the remaining 19\%, 17\% are not accessible by anatomical search terms. Approximately $2 \%$ of the searches will retrieve false positives, that is genes which are not expressed in the tissue being queried. Based on these results, the core set of 29 terms was used in a pilot study to evaluate further the feasibility of the candidate gene selection procedure.

\section{Database Search by Syndrome Patterns}

In order to test the feasibility of using expression patterns for the selection of candidate genes, we first examined seven syndromes for which the causative gene is known. In four of these, the disease gene was identified as one of 19 to 45 candidate genes (Table 3 ). In the other three cases, the gene was not found by using the selected combination of search terms. The $T B X 5$ gene for Holt-Oram syndrome was retrieved by using 'limb' as a search term, but not by 'heart'. Alternative search terms were used. Queries using 'cardiac', 'ventricle' and 'atrium' were raised, all with negative results. Similarly, the Jag2 gene was not identified for Alagille syndrome, since only the 'heart' query was positive, but not 'eye' or 'liver'. The gene for Townes-Brocks syndrome (HSAL1 or SSAL) did not feature at all in the GXD, presumably because the mouse homologue has not been identified. Thus, our analysis of seven multiple congenital anomaly syndromes identified the causative gene in the majority, but also showed up some of the weaknesses of this system using current databases such as GXD.

We examined a larger series of syndromes for which the genes were not yet known in order to gain a better view of whether candidate gene lists are specific for

Table 3 Result of search with syndromes of known genesis

\begin{tabular}{llll}
\hline Disorder/MIM number & Search terms & Causative gene identified? & Selectivity \\
\hline Tricho-Dento-Osseous & Ectoderm Skeleton Tooth & yes; DLX3 & 1/19 \\
Ulnar Mammary & Limb Mammary & yes; TBX3 & $1 / 22$ \\
Townes-Brocks & Limb Ear & no; HASL1 & NA \\
Holt-Oram & Limb Heart & no; TBX5 & NA \\
Hand-Foot-genital & Limb Genital & yes; HOXA13 & yes; PAX2 \\
Renal-Coloboma & Renal Eye & no;Jag2 & $1 / 45$ \\
Alagille & Liver Eye Heart & & NA \\
\hline
\end{tabular}

$\mathrm{NA}=$ no applicable. 
each syndrome description, and whether candidates on these lists are in fact biologically plausible, ie real candidates. Since only 29 terms from the list were used to describe 20 syndromes, the possibility of overlap between syndrome descriptions exists, perhaps causing different syndromes to be described by an identical set of terms. As can be seen in Table 1, this was not the case in the pilot. Thus, a relatively limited number of terms still conferred a unique syndrome 'pattern' in this pilot experiment. The syndrome signatures were used to query the Gene eXpression Database. The gene listings resulting from each query were downloaded and combined to generate a list of candidate genes specific to each syndrome. As expected, different numbers of terms yielded different numbers of candidate genes. For four syndromes, two terms were used to define the characteristic pattern of organ involvement. These searches yielded on average 25 genes per syndrome. For twelve syndromes, three terms were used. The average yield of these searches was 16 genes per syndrome. Four syndromes were described with four terms each. The yield in these searches was four genes per syndrome. Based on these results the pilot was continued with the 12 syndromes that were described using a combination of three blanket terms. A total of 20 different terms was used to describe these 12 syndromes. The total number of candidate genes retrieved from the GXD for the 12 syndromes combined was 115 . This is $7.4 \%$ of the total number of 1552 genes that could have been retrieved by the 20 search terms separately. The number of genes picked up by the searches varied from one (OFD type 3; terms 'tongue', 'digit', 'brain') to 61 (Costello syndrome; terms 'heart', 'brain', 'skin'). For the 115 genes that were retrieved for the 12 syndromes, 57 genes $(49.6 \%)$ were picked up only once and 58 genes $(50.4 \%)$ between two and eight times. An example of a syndrome query is given in Table 4. Here 56 genes were retrieved for the Walker-Warburg syndrome (WWS) and evaluated for their potential as candidate genes. Gene function was assessed using the Mouse Genome Database. ${ }^{17}$ Mouse knockout models were searched in TBase. ${ }^{18}$ The search terms used in individual searches were 'muscle', 'eye' and 'brain'. As shown in Table 3, 15 genes were not considered further as syndrome-specific candidate genes on the basis of their known role as housekeepers or their ubiquitous expression. Another 20 genes are associated with human or mouse phenotypes which do not resemble WWS. Therefore the likelihood of these genes causing WWS is reduced. Among these were 10 mouse knock- out models, illustrating the value of knockouts for the evaluation of candidate genes. This proportion can be expected to increase in future, as more developmentally important genes are targeted in the mouse. Another 11 genes were not likely candidates because their products are known to function in single biochemical pathways that are not likely to explain the syndromic pattern of organ involvement. For example, Isll is a LIM-homeodomain gene required for the development of pancreatic islet cells and motor neurons. ${ }^{19}$ The first analysis left 10 genes which merit further evaluation. Of these, eight are associated with human or mouse phenotypes featuring brain, eye and/or muscle abnormalities. The combination of data on expression patterns and function suggested that the remaining two genes may be candidates for the disorder (Table 3 ). To prioritise between these 11 candidate genes, positional information was sought.

Recently, the muscle-eye-brain disease (MEBD) gene was mapped to human chromosome $1 \mathrm{p} 32 .{ }^{20}$ Whether $M E B D$ is allelic to WWS is not known, but the syndromes have a similar pattern of organ involvement. No positional candidate genes could be identified in this region, so it was of interest to check whether any of the candidate genes identified above maps near this region or is in a syntenic mouse chromosomal region. None of the genes in our list has been localised to the 1 p32 region in humans. However, $C d h 4$, which has not yet been mapped in humans, may be in the relevant area, as it is in a region on mouse chromosome 2 which shows synteny with human 1p32-33 and therefore is an interesting candidate. Cdh4 belongs to the cadherin family of cellular adhesion molecules. It is expressed in muscle, retina and brain; inhibition of cadherin action in the brain leads to neuronal migration defects. ${ }^{21,22}$ In view of its expression pattern, Cdh4 is an attractive candidate gene for this disorder. Thus, the search for Walker-Warburg syndrome uncovered 11 candidate genes, one of which is also a positional candidate for MEBD. Similar results were obtained for the other 11 syndromes in this limited pilot experiment. On average, $23 \%$ of the genes were ubiquitously expressed or housekeeping genes. Of the remainder, six genes (or their associated pathways) were considered potential candidates for one of the syndromes in the pilot study, namely Bmp4, Fgf4, Dct, Mitf, Pdgfra and Lef-1. For these genes, mouse mutants either occur naturally or have been generated, which facilitates their evaluation (Table 5). These are discussed below. A search for DOOR syndrome using 'ear', 'kidney' and 'digit' as 
Table 4 Evaluation of WWS candidate genes

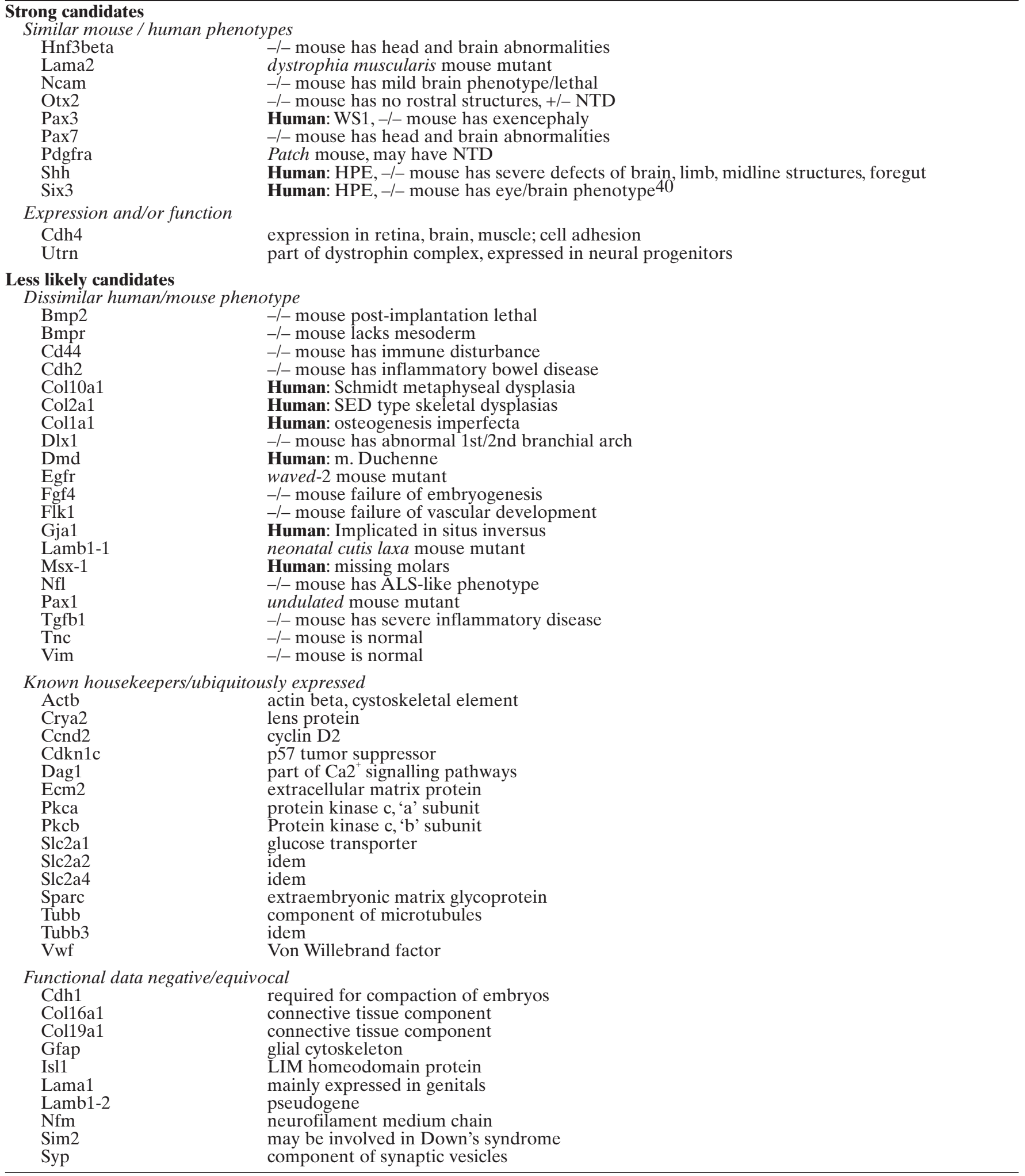

NTD: neural tube defect; HPE: holoprosencephaly; WS1: Waardenburg syndrome type 1; SED: spondyloepiphyseal dysplasia; ALS: amyotrophic lateral sclerosis. 
Table 5 Potential candidate genes found for some of the syndromes described by three terms

\begin{tabular}{lll}
\hline Syndrome & Candidate genes & Arguments for gene \\
\hline $\begin{array}{l}\text { C-syndrome } \\
\text { terms: cranium, digit, brain }\end{array}$ & Fgft & Overexpression in mouse gives cranial and limb abnormalities \\
$\begin{array}{c}\text { Oculocerebral hypopigmentation syndrome } \\
\text { terms: brain, eye, pigment }\end{array}$ & $D c t$ & $\begin{array}{l}\text { Slaty mouse has similar pigment disturbances; Dct expressed } \\
\text { in brain. Involved in pigmentation and eye development, }\end{array}$ \\
& $\begin{array}{l}\text { Mtf } \\
\text { Pdgfra }\end{array}$ & $\begin{array}{l}\text { Pardenburg syndrome type II in humans. } \\
\text { Patched mouse has CNS and pigment anomalies }\end{array}$ \\
$\begin{array}{l}\begin{array}{c}\text { DOOR syndrome } \\
\text { terms: ear, kidney, digit }\end{array} \\
\begin{array}{c}\text { Scalp-ear-nipple syndrome } \\
\text { terms: skin, ear, mammary }\end{array}\end{array}$ & Bmp4 & $-/+$ mouse has polydactyly, cystic kidneys and inner ear defects \\
& Lef1 & $-/-$ mouse lacks mammary tissue, hair and teeth
\end{tabular}

terms uncovered five candidate genes, of which $B M P 4$ may be especially convincing. The heterozygous $B m p 4$ knockout mouse has pre-axial polydactyly, cystic kidney malformations and in a number of cases shows circling behaviour consistent with an inner ear defect. Shortening of the nasal and frontal bones, giving the appearance of a boxer's nose, were also noted. ${ }^{23}$ DOOR syndrome is a disorder characterised by onychodystrophy, triphalangeal thumbs, mental retardation, seizures and sensorineural deafness. Cystic malformations of kidneys and urinary tract have also been described. ${ }^{24}$ The face is characterised by a short, broad nose. There is no mention of dystrophic claws or seizures in the knockout mice, but the mouse phenotype seems to resemble the human DOOR phenotype sufficiently to warrant consideration of Bmp4 as a serious candidate gene for this disorder.

One search also resulted in Fgf4 as a candidate gene for $\mathrm{C}$ syndrome (trigonocephaly and polydactyly). An insertional mutation in the mouse upregulating the expression of $F g f 3$ and $F g f 4$ in the mouse causes craniofacial malformations. $^{25}$ The mutant is called Bulgy eye (Bey). Bey mice are viable and fertile but show facial shortening with increased interorbital distance and craniosynostosis. According to Carlton et al, who described the mutant, the Bey mutation resembles Crouzon syndrome. ${ }^{25}$ Although two $F g f$ s are upregulated here, this study suggests that $F g f 4$ may be involved in the regulation of suture closure. Fgf4 is involved in limb patterning as well. In the Strong's luxoid mouse mutant, ectopic expression of $\mathrm{Fgf4}$ causes polydactyly. ${ }^{26} \mathrm{Fgf4}$ is normally secreted by the ZPA and is one of the regulators of antero-posterior polarity in the limb. ${ }^{27}$ These data suggest that either Fgf4 or a gene regulating its expression in the developing skull and limbs may be involved in $\mathrm{C}$ syndrome, which is characterised by trigonocephaly and polydactyly. ${ }^{28}$ For three other syndromes in the list, potential candidate genes were also obtained. Various mutations in dopachrome tautomerase, $D c t$, have been found in the Slaty mouse mutant, which has patchy to diffuse hypopigmentation. ${ }^{29}$ This phenotype models the pigmentation phenotype of oculocerebral hypopigmentation syndrome. Moreover, Dct is also expressed in the brain although its role in that organ is as yet unclear. ${ }^{29}$ Mutations in the Mitf gene cause microphthalmia in the mouse and are responsible in humans for about $15 \%$ of Waardenburg syndrome type II cases. ${ }^{30}$ In the mouse, a number of Mitf alleles exist, all associating disturbances of pigmentation with various other abnormalities. ${ }^{31}$ This suggests that Mitf may be relevant for a number of different pigmentation phenotypes in humans. As such this gene is a candidate for oculocerebral hypopigmentation syndrome in which microphthalmia has been reported to occur. ${ }^{32}$ Pdgfra is mutated in the Patch mouse, that shows patchy depigmentation ${ }^{33}$ and, depending on its genetic background, other anomalies varying from cardiovascular malformations to neural tube defects. ${ }^{34}$ Although neural tube defects are not a feature of oculocerebral hypopigmentation syndrome, the mouse appears to be more prone to developing neural tube defects upon perturbation of developmental genes and it is conceivable that a similar mutation in humans could give rise to a more subtle neural tube phenotype. Finally, the search for the scalp-ear-nipple syndrome produced Lef-1 as a candidate. Scalp-earnipple syndrome is characterised by hairless nodules on the scalp, malformed external ears, hypo- to aplasia of nipples and breast tissue and widely spaced or missing secondary teeth. ${ }^{35}$ Lymphoid enhancer factor-1 is an HMG-domain DNA-binding protein expressed in the neural crest, mesencephalon, tooth germs and other sites during embryogenesis. ${ }^{36}$ It is a component of the Wnt pathway, and probably modulates the actions of $\beta$-catenin. ${ }^{37}$ Homozygous deficiency of this gene causes postnatal lethality in mice. Mutant mice lack teeth, 
mammary glands, whiskers and hair. All anlagen of these tissues are reduced in number and are arrested in their development. Although the phenotype is lethal in mice and nodules of the skin were not described, the lack of hair, missing teeth and aplasia of breast tissue suggest that Lef-1 may be a candidate gene for scalpear-nipple syndrome.

\section{Discussion}

If it is to be used for the purpose of generating positionindependent candidate genes, a gene expression database should ideally have the following features:

- include all human genes;

- be updated regularly;

- all genes in the database should be examined for expression in a large number of tissues, so that both presence and absence of expression are listed;

- contain data in the form of gene expression patterns for defined embryonal stages, determined by comparable, standardised methods;

- the database should allow searching with uniform search terms for genes, tissues, and stages of development.

It appears that the GXD at present fails to fulfil several of these requirements.

At the time our study was undertaken, the GXD contained less than $3 \%$ of the total number of genes expected to be present in the mouse. Often, only a few tissues have been examined for gene expression patterns, so there is no way of knowing whether the data presented accurately reflect the expression pattern of a gene. At the time of our study no standard terminology had been implemented in the GXD to describe expression patterns, which often necessitates multiple searches with synonyms for a single tissue. In addition, a limited number of errors are probably inevitable in the construction of this and similar large databases. On average, $2 \%$ of all query results will not be associated with verifiable expression data and hence constitute a false positive result.

Although it is possible to search for stage-specific gene expression patterns, stage grouping in the GXD database was not very precise. Embryonic stages are described as days post coitum (dpc) instead of embryonic Theiler stages. Since dpc sometimes cover several
Theiler stages and embryonic tissues sometimes appear over the course of one Theiler stage, this is a source of potential error. Lastly, gene expression patterns listed in the GXD had been determined by a number of different methods. Comparing expression data for various candidate genes and assessing the reliability of these data is not yet optimal. It may be argued that there are examples of mutations in genes that are ubiquitously expressed, but cause a very specific pattern of organ involvement. Examples are eye diseases such as RP3 and gyrate atrophy which are due to mutations in ubiquitously expressed genes. This does not in itself invalidate the approach outlined here. In fact, a general feature of all candidate gene strategies is that they represent educated guesses and hence should be regarded as hypothesis-generating rather than as problem-solving.

It is of interest to note that out of seven syndromes for which the gene is known, a search based on organ involvement returned a list of genes which included the real disease gene in four of seven cases (trichodentoosseous, hand-foot-genital, unlar-mammary, and renal-coloboma syndromes, Table 3 ). In addition, our limited pilot experiment of 20 syndromes of unknown genesis yielded credible candidate genes for several of these (Table 5). Therefore, even though many problems still have to be solved, it would appear to be possible to use simple search terms that describe organs or anatomical regions, by which one can convert human malformation syndromes into a syndrome-specific developmental gene expression pattern. Using only 29 simple terms at present covers $65 \%$ of the GXD and a manageable list of 153 terms covers $84 \%$. Thus, the number of terms that is required to translate a syndrome to an expression pattern is not large.

As the number of genes in expression databases increases, so will the numbers retrieved in this type of search. Therefore, secondary criteria for the ranking of candidates will have to be developed. For this purpose, genetic localisation would be a useful possibility. Also, the number of genes retrieved will be smaller if more search terms, or specific periods for embryonic gene expression are used to define the syndromes.

In conclusion, our data suggest that it may ultimately be possible to probe gene expression databases for candidate genes for multiple congenital malformation syndromes using simple syndrome descriptions. The potential value of this is illustrated by recent data. ${ }^{38}$ For instance, the genes for Townes-Brocks syndrome (SAL1) was initially identified on the basis of positional 
information. Upon examination of the expression pattern of its mouse homolog (Msal1) it became clear that it matched the pattern of abnormalities observed in the syndrome. This established the SAL1 gene as an excellent candidate for Townes-Brocks syndrome, a hypothesis which was borne out by subsequent mutation analysis. It is also encouraging that in our analysis of seven syndromes due to known gene mutations, we could identify the gene for four of these using expression data as the only clues. If this method is to be used on a larger scale, the expression databases will need to meet as many of the requirements listed above as possible. Projects such as GENEX, an internet-accessible database that integrates mouse anatomy data with gene expression data, ${ }^{39}$ may ameliorate the situation in the near future. In the GENEX database, expression patterns are listed according to Theiler stages. The terms used to describe various tissues and organs were standardised from the beginning. As the authors of the database are also responsible for the expression studies, uniform methodology is assured. When this and other similar databases for human developmental data are fully operational, most of the above requirements will be met. With the expected accumulation of genomics/ proteomics data, these and other databases can be used to identify position-independent candidate genes for human congenital malformation syndromes. Such candidate genes can be the starting point for further investigations. In most cases these will be in the form of targeted linkage analyses in small families. In cases where such family material is unavailable, direct mutation analysis may be the strategy of choice.

\section{Acknowledgements}

This work was supported by the following foundations: Stichting Irene Kinderziekenhuis, Stichting Het Gehandicapte Kind and Stichting De Drie Lichten (grants to HB and HvB).

\section{References}

1 Friedman PA, Kaufman S, Kang ES: Nature of the molecular defect in phenylketonuria and hyperphenylalaninaemia. Nature 1972; 240: 157-159.

2 Fernandez-Canon JM, Granadino B, Beltran-Valero de Bernabe D et al: The molecular basis of alkaptonuria. Nat Genet 1996; 14: 19-24.

3 Wood WI, Capon DJ, Simonsen CC et al: Expression of active human factor VIII from recombinant DNA clones. Nature 1984; 310: 330-337.
4 Ballabio A: The rise and fall of positional cloning? Nat Genet 1993; 3: 277-279.

5 Beighton P, De Paepe A, Hall JG et al: Molecular nosology of heritable disorders of connective tissue. Am J Med Genet 1992; 42: 431-448.

6 Dryja TP: Gene-based approach to human gene-phenotype correlations. Proc Natl Acad Sci USA 1997; 94: 12117-12121.

7 Robertson NG, Skvorak AB, Yin Y et al: Mapping and characterization of a novel cochlear gene in human and in mouse: a positional candidate gene for a deafness disorder, DFNA9. Genomics 1997; 46: 345-354.

8 Favor J, Sandulache R, Neuhäuser-Klaus A et al: The mouse Pax $2^{1 \mathrm{Neu}}$ mutation is identical to a human PAX2 mutation in a family with renal-coloboma syndrome and results in developmental defects in brain, ear, eye and kidney. Proc Natl Acad Sci USA 1996; 93: 13870-13875.

9 Sanyanusin P, Schimmenti LA, McNoe LA et al: Mutation of the PAX2 gene in a family with optic nerve colobomas, renal anomalies and vesico-ureteral reflux. Nat Genet 1995; 9: 358-364.

10 Winter RM, Baraitser M: The London Dysmorphology Database: a Computerized Database for the Diagnosis of Rare Dysmorphic Syndromes. 5th edn. Oxford University Press: Oxford, 1998.

11 Preus M, Ayme S: Formal analysis of dysmorphism: objective methods of syndrome definition. Clin Genet 1983; 23: 1-16.

12 Bankier A, Keith CG: POSSUM: the microcomputer laser-videodisc syndrome information system. Ophtalm Paediatr Genet 1989; 10: 51-52.

13 Bankier A, Sillence DM, Maroteaux P et al: 'OSSUM', an illustrated database of skeletal dysplasias. 1994. Possum s.o Telemedia Software Laboratories http://tsl.cpg.com.au/ possum5.htm

14 Van Steensel MAM, Winter RM: Internet databases for clinical geneticists - an overview. Clin Genet 1998; 53: 323-330.

15 The scripts used in the pilot as well as the word list may be accessed and downloaded at http://baserv.uci.kun.nl/ $\sim$ mvanstee/pilot.htm

16 Online Mendelian Inheritance in Man. URL: http:/ /www.ncbi.nlm.nih.gov/omim

17 Mouse Genome Database. URL: http://www.informatics.jax.org/mgd.html

18 Transgenic/Targeted mutation database. URL: http:/tbase.jax.org/

19 Thor S, Ericson J, Brannstrom T, Edlund T: The homeodomain LIM protein Isl-1 is expressed in subsets of neurons and endocrine cells in the adult rat. Neuron 1991; 7: 881-889.

20 Avela K, Pihko H, Santavuori P, Talim B: Assignment of the muscle-eye-brain (MEB) disease gene to $1 \mathrm{p} 32$ by linkage analysis. Am J Hum Genet 1998; 63(S): A285.

21 Hutton JC, Christofori G, Chi WY et al: Molecular cloning of mouse pancreatic islet R-cadherin: differential expression in endocrine and exocrine tissue. Mol Endocrinol 1993; 7: 1151-1160.

22 Takeichi M, Inuzuka H, Shimamura K, Matsunaga M, Nose A: Cadherin-mediated cell-cell adhesion and neurogenesis. Neurosci Res 1990; 13 (Suppl): S92-96. 
23 Dunn NR, Winnier G, Hargett LK, Schrick JJ, Fogo AB, Hogan BLM: Haploinsufficient phenotypes in bmp4 heterozygous null mice and modification by mutations in gli3 and alx4. Dev Biol 1997; 188: 235-247.

24 Thornton CM, Magee AC, Thomas PS, Feakins R, Nevin NC, O'Hara MD: Congenital heart disease and urinary tract abnormalities in two siblings with DOOR syndrome. Paediatr Pathol 1994; 14: 797-803.

25 Carlton MB, Colledge WH, Evans MJ: Crouzon-like craniofacial dysmorphology in the mouse is caused by an insertional mutation at the Fgf3/Fgf4 locus. Dev Dyn 1998; 212: 242-249.

$26 \mathrm{Qu} \mathrm{SH}$, Tucker SC, Ehrlich JS et al: Mutations in mouse Aristaless-like4 cause Strong's luxoid polydactyly. Development 1998; 125: 2711-2721.

27 Johnson JR, Tabin C: Molecular models for verebtrate limb development. Cell 1997; 90: 979-990.

28 Opitz JM, Johnson RC, McCreadie SR, Smith DW: The C syndrome of multiple congenital anomalies. Birth Defects Orig Art Ser 1969; 5(2): 161-166.

29 Budd PS, Jackson IJ: Structure of the mouse tyrosinaserelated protein-2/dopachrome tautomerase (Tyrp2/Dct) gene and sequence of two novel slaty alleles. Genomics 1995; 29: 35-43.

30 Tassabehji M, Newton VE, Read AP: Waardenburg syndrome type 2 caused by mutations in the human microphthalmia (MITF) gene. Nat Genet 1994; 8: 251-255.

31 Amiel J, Watkin PM, Tassabehji M, Read AP, Winter RM: Mutation of the MITF gene in albinism-deafness syndrome (Tietz syndrome). Clin Dysmorphol 1998; 7: $17-20$.
32 Cross HE, McKusick VA, Breen W: A new oculocerebral syndrome with hypopigmentation. J Pediatr 1967; 70: 398-406.

33 Stephenson DA, Mercola M, Anderson E et al: Plateletderived growth factor receptor alpha-subunit gene (Pdgfra) is deleted in the mouse patch $(\mathrm{Ph})$ mutation. Proc Natl Acad Sci USA 1991; 88: 6-10.

34 Payne J, Shibasaki F, Mercola M: Spina bifida occulta in homozygous Patch mouse embryos. Dev Dyn 1997; 209: 105-116.

35 Edwards MJ, McDonald D, Moore P, Rae J: Scalp-earnipple syndrome: additional manifestations. Am J Med Genet 1994; 50: 247-250.

36 Van Genderen C, Okamura RM, Farinas I et al: Development of several organs that require inductive epithelialmesenchymal interactions is impaired in LEF-1-deficient mice. Genes Dev 1994; 8: 2691-2703.

37 Moon RT, Brown JD, Torres M: WNTs modulate cell fate and behavior during vertebrate development. Trends Genet 1997; 13: 157-162.

38 Kohlhase J, Wischermann A, Reichenbach $\mathrm{H}$ et al: Mutations in the SALL1 putative transcription factor gene cause Townes-Brocks syndrome. Nat Genet 1998; 18: 81-83.

39 Bard JBL, Kaufman MH, Dubreuil C et al: An internetaccessible database of mouse developmental anatomy based on a systematic nomenclature. Mech Dev 1998; 74: 111-120.

40 Wallis DE, Roessler E, Hehr U et al: Missense mutations in the homeodomain of SIX3 are associated with holoprosencephaly. Am J Hum Genet 1998; 63S: A27. 